\title{
Арочная пластика левого предсердия при коррекции митрального порока
}

\author{
В. В. Попов, А. А. Большак, В. В. Лазоришинец
}

Национальный институт сердечно-сосудистой хирургии имени Н. М. Амосова, г. Киев

\section{The arch-like plasty of the left atrium in correction of a mitral valve failure}

\author{
V. V. Popov, A. A. Bolshak, V. V. Lazoryshynets
}

Amosov National Institute of Cardiovascular Surgery, Kyiv

\section{Реферат}

Цель. Изучение возможностей методики арочной пластики левого предсердия при коррекции митрального порока в сочетании с дилатацией левого предсердия.

Материалы и методы. В анализ включены результаты хирургического лечения 190 пациентов с митральным пороком в сочетании с дилатацией левого предсердия, которые были оперированы в Национальном институте сердечнососудистой хирургии имени Н. М. Амосова НАМН Украины за период с 01.01.2012 до 01.01.2021 г. Основную группу составили 103 пациента, которым выполнена коррекция митрального порока в сочетании с оригинальной методикой арочной пластики левого предсердия. В контрольную группу вошли 87 пациентов, которым выполнена коррекция митрального порока без сопутствующей пластики левого предсердия.

Результаты. Из 103 оперированных пациентов основной группы на госпитальном этапе умер 1 (летальность 0,9\%). Динамика эхокардиографических показателей на этапах лечения: конечно-систолический индекс левого желудочка - (63,1 $\pm 11,3)$ мл/м² (до операции), $(58,3 \pm 8,4)$ мл/м² (после операции), $(49,4 \pm 9,2)$ мл/м² (отдаленный период); фрак-

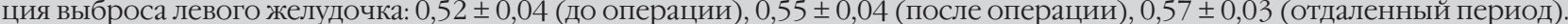
Диаметр левого предсердия: $(58,8 \pm 6,4)$ мм (до операции), $(41,4 \pm 5,3)$ мм (после операции), $(43,9 \pm 2,3)$ мм (отдаленный период). Синусовый ритм в отдаленном периоде удерживается у 75 (78,9\%) из 95 пациентов. Из 87 оперированных пациентов контрольной группы умерли 2 (летальность 2,3\%). Динамика эхокардиографических показателей на этапах лечения: конечно-систолический индекс левого желудочка- $(67,3 \pm 11,3)$ мл/м² (до операции), $(60,4 \pm 9,3)$ мл/

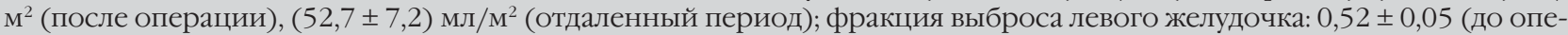
рации), 0,54 \pm 0,05 (после операции), 0,54 \pm 0,03 (отдаленный период). Диаметр левого предсердия: (59,5 $\pm 2,3)$ мм (до операции), $(57,5 \pm 3,7)$ мм (после операции), $(68,5 \pm 3,4)$ мм (отдаленный период). Синусовый ритм удерживается у 18 $(22,5 \%)$ из 80 пациентов, прослеженных в отдаленном периоде.

Выводы. Арочная пластика левого предсердия малотравматичная и эффективная процедура, приводящая к значительному улучшению морфометрии левого предсердия и сопровождающаяся низким риском послеоперационной летальности

Ключевые слова: дилатация левого предсердия; арочная пластика левого предсердия; протезирование митрального клапана; пластика митрального клапана; искусственное кровообращение.

\section{Abstract}

Objective. Studying the possibilities of the method of the left atrium arch-like plasty while correcting of a mitral failure in combination with the left atrium dilatation.

Materials and methods. Into the analysis of the surgical treatment results in 190 patients, suffering mitral failure in combination with the left atrium dilatation, who were operated in the National Institute of Cardio-Vascular Surgery named after N. M. Amosov NAMS of Ukraine in a period from 01.01.2012 to 01.01.2021 yr, were included. The main group consisted of 103 patients, to whom correction of a mitral failure in combination with original procedure of the arch-like plasty of left atrium was performed. Into a control group 87 patients were included, to whom the correction of a mitral valve failure was done without concomitant plasty of left atrium.

Results. Of 103 operated patients of the main group on the hospital stage 1 have died (0.9\% lethality). Dynamics of echocardiographic indices on the treatment stages was following: definitely-systolic index of the left ventricle $-(63.1 \pm 11.3) \mathrm{ml} / \mathrm{m}^{2}$ (preoperatively), $(58.3 \pm 8.4) \mathrm{ml} / \mathrm{m}^{2}$ (postoperatively), $(49.4 \pm 9.2) \mathrm{ml} / \mathrm{m}^{2}$ (remote period); the left ventricle ejection fraction: $0.52 \pm 0.04$ (preoperatively), $0.55 \pm 0.04$ (postoperatively), $0.57 \pm 0.03$ (remote period). Diameter of left atrium: (58.8 \pm 6.4$) \mathrm{mm}$ (preoperatively), $(41.4 \pm 5.3) \mathrm{mm}$ (postoperatively), $(43.9 \pm 2.3) \mathrm{mm}$ (remote period). Sinus rhythm in a remote period was stable in 75 (78.9\%) of 95 patients. Of 87 operated patients of a control group 2 died (lethality $2.3 \%$ ). Dynamics of the echocardiographic indices on the treatment stages was following: definitely-systolic index of left ventricle- $(67.3 \pm 11.3) \mathrm{ml} / \mathrm{m}^{2}(\mathrm{preoper}-$ atively), $(60.4 \pm 9.3) \mathrm{ml} / \mathrm{m}^{2}$ (postoperatively), $(52.7 \pm 7.2) \mathrm{ml} / \mathrm{m}^{2}$ (remote period); the left ventricle ejection fraction: $0.52 \pm 0.05$ (preoperatively), $0.54 \pm 0.05$ (postoperatively), $0.54 \pm 0.03$ (remote period). Diameter of left atrium: $(59.5 \pm 2.3) \mathrm{mm}$ (preoperatively), $(57.5 \pm 3.7) \mathrm{mm}$ (postoperatively), $(68.5 \pm 3.4) \mathrm{mm}$ (in remote period). Sinus rhythm was stable in $18(22.5 \%)$ of $80 \mathrm{pa}-$ tients, followed in the remote period.

Conclusion. The arch-like plasty of left atrium is a low-traumatic and effective procedure, leading to significant improvement of the left atrium morphometry and accompanied by low risk for postoperative lethality.

Keywords: dilatation of left atrium; arch-like plasty of left atrium; prosthesis of mitral valve; plasty of mitral valve; artificial blood circulation. 
Дилатация левого предсердия (ДЛП) является клинически значимым фактором риска хирургического лечения изолированного митрального порока сердца и встречается у 21 - 39\% больных [1 - 4]. При ДлП зачастую происходит сдавление задне-базального сегмента левого желудочка (ЛЖ), кроме того, подвергаются компрессии нижняя и средняя доли правого легкого и главного бронха слева. Указанные нарушения определяют значительную сердечно-дыхательную недостаточность [2, 5 - 8]. В отдаленном послеоперационном периоде длП ведет к прогрессированию проявлений сердечно-дыхательной недостаточности, повышенному риску тромбоэмболических осложнений [1, 6, 9 - 11]. ДЛП препятствует восстановлению синусового ритма как на госпитальном этапе, так и в отдаленные сроки [1, 4, 10, 12 - 14].

Принимая во внимание, что изолированная коррекция митрального порока при наличии сопутствующей ДлП ведет к последующему расширению левого предсердия (ЛП) в отдаленном периоде, целесообразно уменьшение ЛП хирургическим путем [13 - 16].

Существуют различные способы уменьшения диаметра ЛП. Однако, невзирая на различные предложенные варианты редукции ЛП, не ослабевает интерес к изучению данной категории пациентов $[1,5,17,18]$.

Нами предложена коррекция митрального порока в сочетании с оригинальной методикой арочной пластики ЛП. Данная процедура проявила себя как малотравматичная и эффективная. В то же время она позволила существенно улучшить морфометрию ЛП, удержать синусовый ритм, снизив уровень тромбоэмболических осложнений в послеоперационном периоде. Кроме того, она сопровождается низким риском госпитальной летальности и специфических осложнений и является желательной процедурой для коррекции длП.

Цель исследования: изучение возможностей методики арочной пластики ЛП при коррекции митрального порока в сочетании с ДЛП.

\section{Материалы и методы исследования}

В анализ включены данные 190 пациентов с изолированным митральным пороком и длп, оперированных в Национальном институте сердечно-сосудистой хирургии имени Н. М. Амосова НАМН Украины с 1 января 2012 до 1 января 2021 г. У всех пациентов диагностирован порок митрального клапана, требующий хирургической коррекции, в сочетании с ДЛП. Ведущей этиологической причиной поражения клапана был ревматизм в сочетании с липоидозом.

Средний возраст оперированных составил $(55,8 \pm 7,7)$ года. Женщин было 106 (55,8\%), мужчин - 84 (44,2\%). Распределение пациентов в зависимости от исходного функционального класса (ФК) по Нью-Йоркской классификации сердечной недостаточности ( New York Heart Association Functional Classificattion - NYHA) было следующим: III ФК - 85 (44,8\%), IV ФК - 105 (55,2\%). Фибрилляция предсердий (ФП) отмечена у всех пациентов. Пациенты были разделены на две группы: основную (n=103), пациентам этой группы выполняли коррекцию ДЛП при коррекции митрального порока, и контрольную (n=87), пациентам этой группы вмешательство на ЛП не выполняли.

Дооперационные зхокардиографические показатели в основной группе: конечно-систолический индекс Лж - $(63,1 \pm 11,3)$ мл/м²; фракция выброса ЛЖ - 0,52 \pm 0,04. Диаметр ЛП составил $(58,8 \pm 6,4)$ мм. Дооперационные эхокардиографические показатели в контрольной группе: конечно-систолический индекс ЛЖ- $(67,3 \pm 11,3)$ мл/ $\mathrm{M}^{2}$; фракция выброса ЛЖ - 0,52 \pm 0,05. Диаметр ЛП составил $(59,5 \pm 2)$ мм. По приведенным показателям основная и контрольная группы статистически значимо не отличались ( $>$ > 0,05).

Диагностику ДлП проводили также при помощи компьютерной томографии (рис. 1).

Операции выполняли в условиях искусственного кровообращения и умеренной гипотермии $\left(28-32^{\circ} \mathrm{C}\right)$. Защита миокарда осуществлялась в условиях ретроградной, анте- и ретроградной фармакохолодовой кардиоплегии (кустодиол) в сочетании с наружным охлаждением. Доступ к митральному клапану осуществлялся через ЛП позади и параллельно межпредсердной борозде. В митральную позицию имплантировались механические двустворчатые протезы St. Jude Medical, ATS, On-X, Carbomedics, Edwards-Mira (n=162), биопротезы (n=5), а также использовались опорные кольца для пластики $(\mathrm{n}=23)$.

Протезы фиксировали отдельными П-образными швами с тефлоновыми прокладками в количестве 13 - 18. Митральные протезы имплантировали в промежуточную позицию. Операция фрагментации ЛП для восстановления синусового ритма выполнена у 33 (17,3\%) пациентов. У 7 (3,7\%) пациентов выявлен массивный тромбоз ЛП (уровень отложения тромботических масс не менее $1 / 3$ объ-

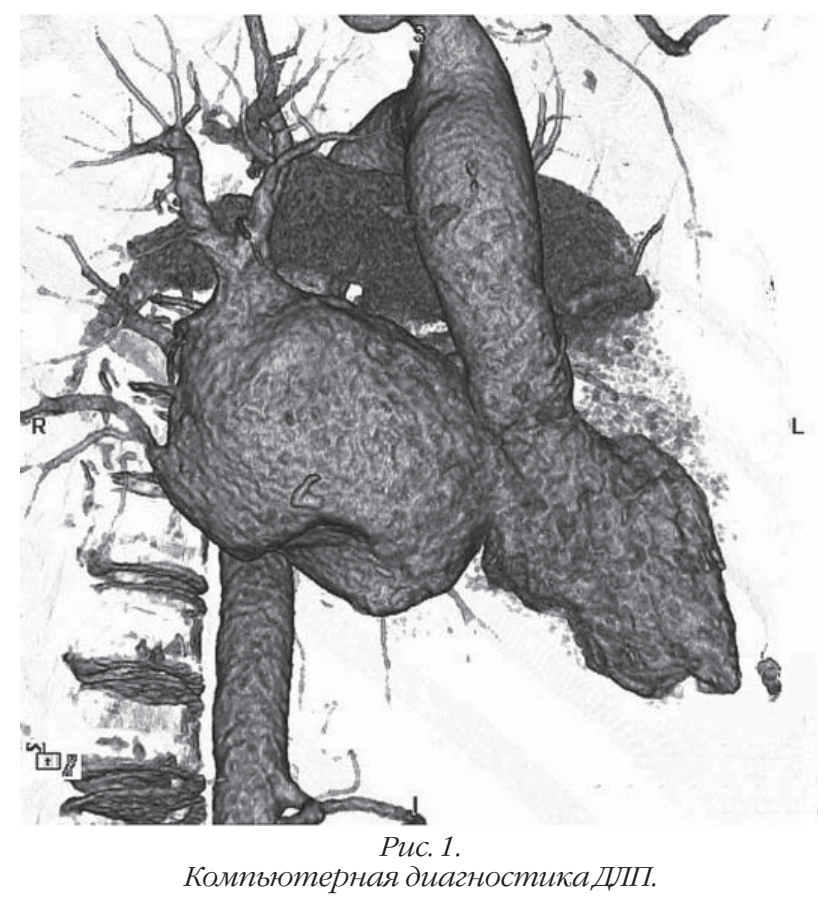




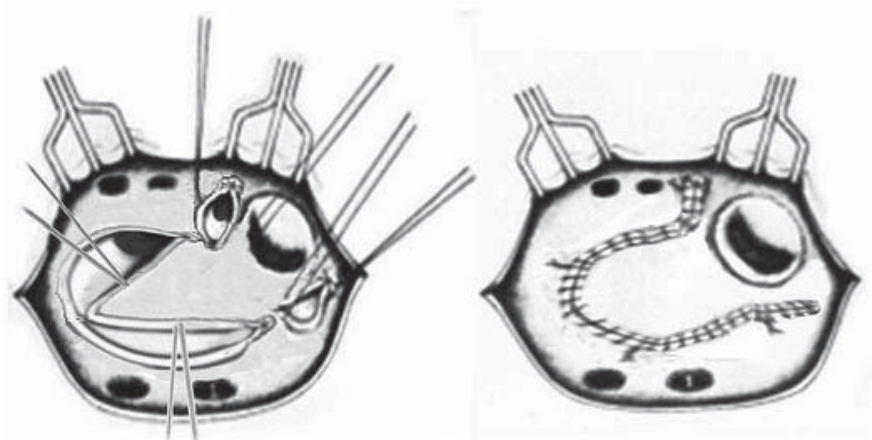

Puc.2.

Арочная пластикалП.

ема ЛП, не считая ушка). Из исследования исключены пациенты с сопутствующим аортальным, трикуспидальным пороком, аорто-коронарным шунтированием.

Арочная пластика ЛП разработана и внедрена профессором В. В. Поповым в январе 2012 г. Идеология пластики направлена на максимальную редукцию ЛП, чтобы восстановить правильный ритм. Арочную пластику ЛП начинали перевязыванием снаружи с последующей резекцией ушка ЛП и выполняли следующим образом. Первый этап - парааннулярное плицирование задней стенки ЛП в двух местах с разрывом пространства по середине. Затем по сходящимся линиям от мест парааннулярной краевой фиксации были плицированы 2 участка ЛП между правыми и левыми легочными венами, которые соединялись на вершине между собой, завершая формирование арки. Для пластики использовалась нить prolene 3-0 (рис. 2).

Технических проблем при выполнении коррекции не было. Продолжительность пережатия аорты в основной группе составила $(86,9 \pm 13,5)$ мин, в контрольной группе - $(62,6 \pm 10,3)$ мин $(\mathrm{p}<0,05)$. На госпитальном этапе осложнений, связанных с методикой выполнения операции, в основной группе не отмечено.

\section{Результаты}

В основной группе на госпитальном этапе умер 1 пациент (госпитальная летальность составила 0,9\%), причиной смерти явилась пневмония. Инотропная поддерж-

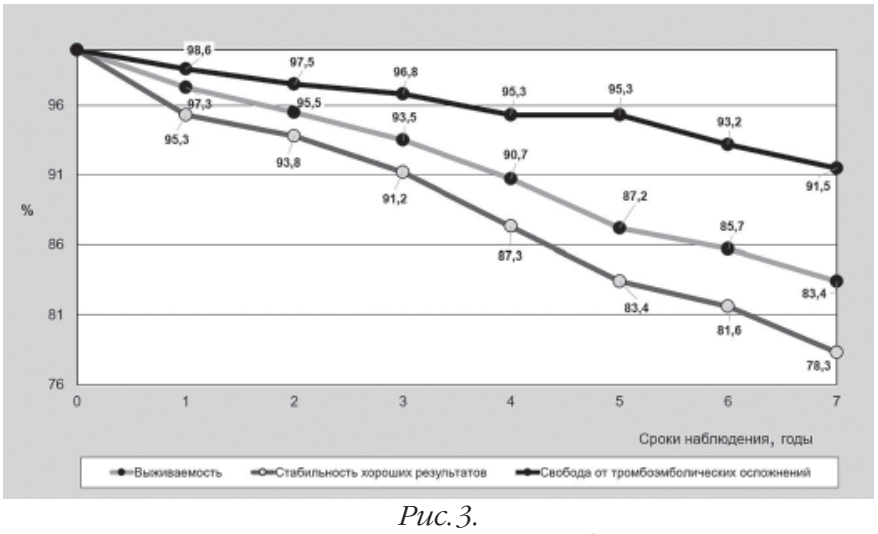

Актуарные кривые выживаемости, стабильности хороших результатов, свободы от тромбоэмболических осложнений в основной группе $(n=103)$. ка (добутамин) составляла в пределах $2-3$ мкг /кг минн в течение первых 72 ч. Пациенты были выписаны на (9,6 $\pm 2,1)$ дня после операции без клинически значимых осложнений. Кровопотеря на госпитальном этапе не превышала 350,0 мл, в связи с чем у 33 (32,0\%) пациентов не использовали компоненты донорской крови на протяжении всего послеоперационного периода.

Динамика эхокардиографических показателей у пациентов основной группы: конечно-систолический индекс лЖ - $(63,1 \pm 11,3)$ мл/м² (до операции), $(58,3 \pm 8,4)$ мл $/$ м $^{2}$ (после операции), (49,4 $\pm 9,2)$ мл/м² (отдаленный период);

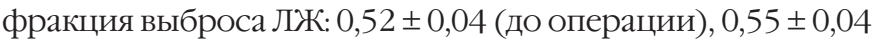
(после операции), 0,57 0,03 (отдаленный период). Диаметр ЛП составил: $(58,8 \pm 6,4)$ мм (до операции), $(41,4 \pm 5,3)$ мм (после операции), (43,9 $\pm 2,3)$ мм (отдаленный период).

В отдаленном периоде, в среднем $(6,7 \pm 0,9)$ года (от полугода до 8 лет), прослежены результаты 95 пациентов, что составило 93,1\% от числа выписанных пациентов (n=102). Хорошие и удовлетворительные результаты отмечены у 77 (81,1\%), неудовлетворительные - у 10 (10,5\%) пациентов. Неудовлетворительные результаты были обусловлены тахиформами ФП (n=4), гипертонической болезнью $(n=2)$, ишемической болезнью сердца - ИБС $(n=2)$, остаточными явлениями после перенесенного острого нарушения мозгового кровообращения - ОНМК $(\mathrm{n}=2)$. В отдаленном периоде умерли 8 (8,4\%) пациентов. Причинами смерти явились: тромбоэмболия сосудов головного мозга (n=2), гипертонический криз, атеросклероз (n=2), прогрессирующая ИБС $(n=1)$, пневмония $(n=1)$, онкологическое заболевание $(\mathrm{n}=1)$, аритмия $(\mathrm{n}=1)$. Причина смерти 1 больного точно не известна. В целом тромбоэмболические осложнения отмечены у 3 (3,2\%) пациентов: тяжелые ( $\mathrm{n}=1)$, легкие $(\mathrm{n}=1)$, приведшие к смерти $(\mathrm{n}=1)$. Синусовый ритм удерживается у 75 (78,9\%) из 102 пациентов, прослеженных в отдаленном периоде.

Согласно данным, представленным на рис. 3, к 7-му году наблюдения отмечены достаточно стабильные показатели выживаемости, хороших результатов, низкий уровень тромбоэмболических осложнений, свидетельствующие о целесообразности выполнения левой атриопластики.

Из 87 оперированных пациентов контрольной группы на госпитальном этапе умерли 2 (госпитальная летальность составила 2,3\%). Причинами смерти явилась сердечная $(\mathrm{n}=1)$ и полиорганная $(\mathrm{n}=1)$ недостаточность.

Инотропная поддержка (добутамин) составляла в пределах 3 - 4 мкг/кг/мин в течение первых 72 ч. Пациенты были выписаны на $(10,2 \pm 2,1)$ дня после операции без клинически значимых осложнений, но сохранялись умеренная декомпенсация и дыхательная недостаточность. Кровопотеря на госпитальном этапе не превышала 350,0 мл, в связи с чем 13 (14,9\%) пациентов не получали препараты донорской крови на протяжении всего госпитального этапа.

Динамика эхокардиографических показателей у пациентов контрольной группы: конечно-систолический ин-

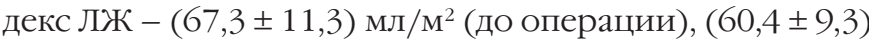


мл/м² (после операции), $(52,7 \pm 7,2)$ мл/м² (отдаленный период); фракция выброса ЛЖ - 0,52 \pm 0,05 (до операции), 0,54 0,05 (после операции), 0,54 \pm 0,03 (отдаленный период). Диаметр ЛП составил: $(59,5 \pm 2,3)$ мм (до операции), (57,5 $\pm 3,7)$ мм (после операции), $(68,5 \pm 3,4)$ мм (отдаленный период).

В контрольной группе в отдаленном периоде, в среднем $(5,6 \pm 2,4)$ года (от полугода до 8 лет), прослежены результаты 80 пациентов, что составило 94,1\% от числа выписанных пациентов ( $\mathrm{n}=85)$. Хорошие и удовлетворительные результаты отмечены у 45 (56,2\%), неудовлетворительные - у 11 (13,7\%) пациентов. Неудовлетворительные результаты были обусловлены остаточными явлениями после перенесенного ОНМК (n=5), тахиформами ФП $(\mathrm{n}=2)$, сердечно-дыхательной недостаточностью $(\mathrm{n}=2)$, ИБС (n=2). Умерли 24 (30,0\%) пациента. Ведущими причинами смерти явились: прогрессирующая сердечно-дыхательная недостаточность ( $=5)$, ОНМК (n=9), тромбоз протеза ( $\mathrm{n}=1)$, гипертонический криз, атеросклероз (n=2), прогрессирующая ИБС $(\mathrm{n}=2)$, аритмия $(\mathrm{n}=1)$, пневмония $(\mathrm{n}=2)$, онкологическое заболевание $(\mathrm{n}=1)$. Причина смерти 1 пациента точно не известна. В целом тромбоэмболические осложнения возникли у 13 (16,3\%) пациентов: тяжелые (n=5), легкие (n=1), приведшие к смерти (n=7).

Синусовый ритм удерживается у 18 (22,5\%) из 80 пациентов, прослеженных в отдаленном периоде.

Актуарные кривые выживаемости, стабильности хороших результатов, свободы от тромбоэмболических осложнений в контрольной группе представлены на рис. 4.

Динамика размеров ЛП в отдаленном периоде представлена на рис. 5.

Как следует из данных, представленных на рис. 5, у пациентов контрольной группы отмечается существенное прогрессирование размеров ЛП.

\section{Обсуждение}

При коррекции митрального порока фактор ДлП является клинически значимым на госпитальном этапе, но в большей степени в отдаленном периоде. Уровень тромбоэмболических осложнений, как и уровень сердечнолегочной недостаточности, у пациентов без коррекции ДЛП в отдаленные сроки достигает критической величины риска, особенно в сочетании с плохо корригируемой давно существующей ФП. Демпфирующая камера расширенного ЛП при наличии тахиформы ФП, являющейся непременным спутником, невзирая на антикоагулянтную терапию, способствует образованию тромбов в полости ЛП и последующей тромбоэмболии. Нелигированное ушко ЛП также является важной причиной тромбоэмболических осложнений.

По мере прогрессирования дЛП сдавление бронхов, трахеи, задней стенки ЛЖ дилатированным ЛП приводит к прогрессирующей сердечно-дыхательной недостаточности, снижающей выживаемость, стабильность хороших результатов. Это предопределяло худшие результаты отдаленного периода у пациентов контрольной группы.

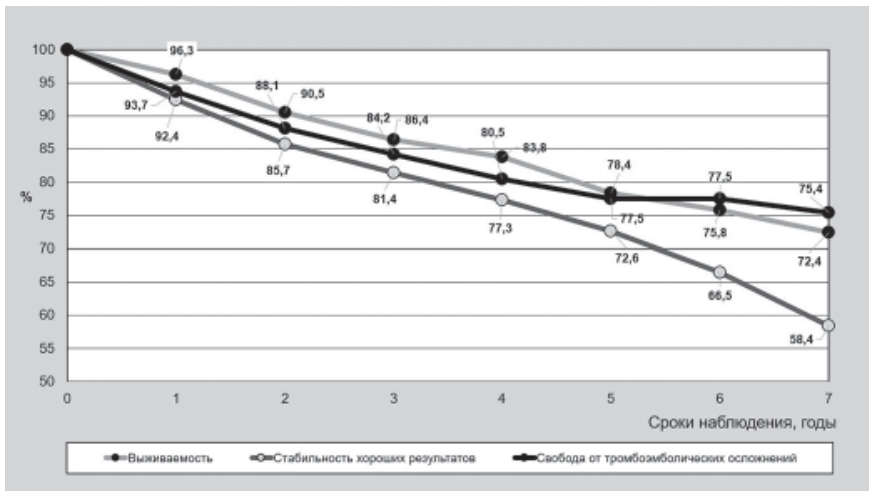

Puc. 4.

Актуарные кривые выживаемости, стабильности хороших результатов, свободы от тромбоэмболических осложнений в контрольной группе $(n=87)$.

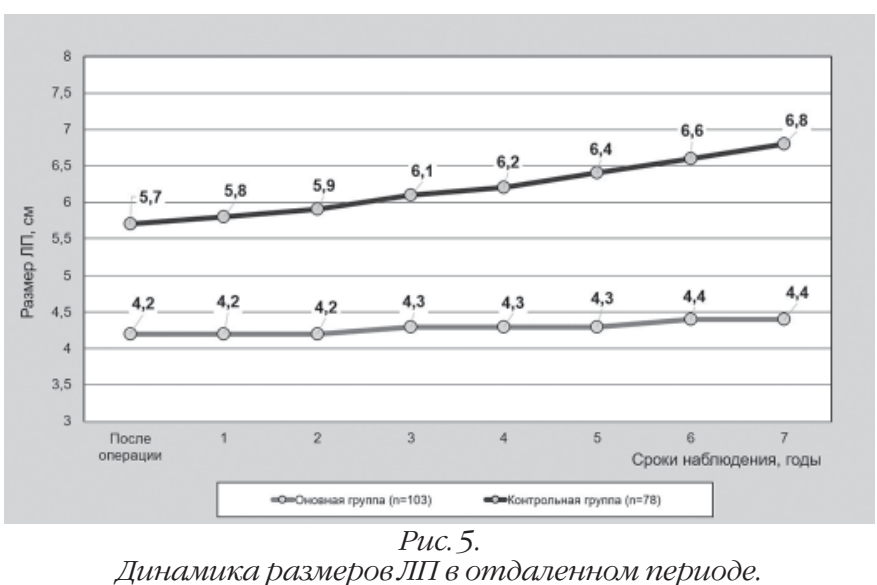

Если результаты лечения в двух группах на госпитальном этапе существенно не отличались, то различия через 5 лет и более после операции становились статистически значимыми. Безусловно, наличие ДлП в пределах 5,5 - 6,0 см в комбинации с ФП является показанием к пластике ЛП и восстановлению правильного ритма, а вариант арочной пластики при лечении митрального порока есть одним из них, который доказал за 9 лет свою целесообразность.

\section{Выводы}

Хирургическое лечение митрального порока в сочетании с предложенной оригинальной методикой арочной пластики-редукции ЛП является желательной процедурой у пациентов с ДЛП (диаметр ЛП 55 - 60 мм). Внедренная методика малотравматичная и эффективная процедура, приводящая к значительному улучшению морфометрии ЛП, сопровождающаяся положительным клиническим эффектом как в ближайшем послеоперационном периоде, так и в отдаленные сроки после оперативного вмешательства.

Финансирование. Бюджетное. Статья является фрагментом научно-исследовательской работы «Розробити та вдосконалити методи хірургічної реконструкції серця при клапанних вадах з атріомегалією». 
Вклад авторов. Попов В. В. - анализ данных, разработка методики; Большак А. А. - сбор, обработка и анализ данных, написание текста; Лазоришинец В. В. - обработка и анализ данных.

Конфликт интересов. Авторы заявляют об отсутствии конфликта интересов.

Согласие на публикацию. Согласие авторов на публикацию статьи получено.

\section{References}

1. Bonow RO, Carabello BA, Chatterjee K, de Leon AC Jr, Faxon DP, Freed $\mathrm{MD}$, et al. ACC/AHA 2006 guidelines for the management of patients with valvular heart disease. J Am Coll Cardiol. 2006 Aug 1;48(3):e1148. doi: 10.1016/j.jacc.2006.05.021. Erratum in: J Am Coll Cardiol. 2007 Mar 6;49(9):1014. PMID: 16875962.

2. Ad N, Damiano RJ Jr, Badhwar V, Calkins H, La Meir M, Nitta T, et al. Expert consensus guidelines: Examining surgical ablation for atrial fibrillation. J Thorac Cardiovasc Surg. 2017 Jun;153(6):1330-54.e1. doi: 10.1016/j.jtcvs.2017.02.027. Epub 2017 Mar 2. PMID: 28390766.

3. La Meir M. Surgical options for treatment of atrial fibrillation. Ann Cardiothorac Surg. 2014 Jan;3(1):30-7. doi: 10.3978/j.issn.2225319X.2014.01.07. PMID: 24516795; PMCID: PMC3904328.

4. Prabhu S, McLellan AJ, Walters TE, Sharma M, Voskoboinik A, Kistler $\mathrm{PM}$. Atrial structure and function and its implications for current and emerging treatments for atrial fibrillation. Prog Cardiovasc Dis. 2015 Sep-Oct;58(2):152-67. doi: 10.1016/j.pcad.2015.08.004. Epub 2015 Aug 4. PMID: 26247494.

5. Masoudi FA, Calkins H, Kavinsky CJ, Slotwiner DJ, Turi ZG, Drozda JP Jr, et al. 2015 ACC/HRS/SCAI Left Atrial Appendage Occlusion Device Societal Overview: A professional societal overview from the American College of Cardiology, Heart Rhythm Society, and Society for Cardiovascular Angiography and Interventions. Catheter Cardiovasc Interv. 2015 Nov;86(5):791-807. doi: 10.1002/ccd.26170. Epub 2015 Sep 21. PMID: 26256562

6. January CT, Wann LS, Alpert JS, Calkins H, Cigarroa JE, Cleveland JC $\mathrm{Jr}$, et al. $2014 \mathrm{AHA} / \mathrm{ACC} / \mathrm{HRS}$ guideline for the management of patients with atrial fibrillation: a report of the American College of Cardiology/ American Heart Association Task Force on Practice Guidelines and the Heart Rhythm Society. J Am Coll Cardiol. 2014 Dec 2;64(21):e1-76. doi: 10.1016/j.jacc.2014.03.022. Epub 2014 Mar 28. Erratum in: J Am Coll Cardiol. 2014 Dec 2;64(21):2305-7. PMID: 24685669.

7. Delling FN, Rong J, Larson MG, Lehman B, Fuller D, Osypiuk E, et al. Evolution of Mitral Valve Prolapse: Insights From the Framingham Heart Study. Circulation. 2016 Apr 26;133(17):1688-95. doi: 10.1161/ CIRCULATIONAHA.115.020621. Epub 2016 Mar 22. PMID: 27006478 ; PMCID: PMC4856536.

8. Weiner MM, Hofer I, Lin HM, Castillo JG, Adams DH, Fischer GW. Relationship among surgical volume, repair quality, and perioperative outcomes for repair of mitral insufficiency in a mitral valve reference center. J Thorac Cardiovasc Surg. 2014 Nov;148(5):2021-6. doi: 10.1016/j.jtcvs.2014.04.040. Epub 2014 Apr 23. PMID: 24836989.
9. Popov VV, Pukas EV. Surgical correction of the mitral valve with plastics of the left atrium in its atriomegaly. Ukrainian Journal of Cardiology. 2018;(Supl 1):132. Russian.

10. Watson DC, Henry WL, Epstein SE, Morrow AG. Effects of operation on left atrial size and the occurence of atrial fibrillation in patients with hypertrophic subaortic stenosis. Circulation. 1977 Jan;55(1):178-81. doi: 10.1161/01.cir.55.1.178. PMID: 576120.

11. Tsang TS, Abhayaratna WP, Barnes ME, Miyasaka Y, Gersh BJ, Bailey $\mathrm{KR}$, et al. Prediction of cardiovascular outcomes with left atrial size: is volume superior to area or diameter? J Am Coll Cardiol. $2006 \mathrm{Mar}$ 7;47(5):1018-23. doi: 10.1016/j.jacc.2005.08.077. Epub 2006 Feb 9. PMID: 16516087.

12. Hashim SW, Pang PY. Antegrade Cardioplegia Decannulation Using the COR-KNOT System in Minimally Invasive Mitral Valve Surgery Innovations (Phila). 2017 Mar/Apr;12(2):150-1. doi: 10.1097/ IMI.0000000000000346. PMID: 28291141.

13. Chao TF, Liu CJ, Liao JN, Wang KL, Lin YJ, Chang SL, et al. Use of Oral Anticoagulants for Stroke Prevention in Patients With Atrial Fibrillation Who Have a History of Intracranial Hemorrhage. Circulation. 2016 Apr 19;133(16):1540-7. doi: 10.1161/CIRCULATIONAHA.115.019794. Epub 2016 Mar 11. PMID: 26969761

14. Lazam S, Vanoverschelde JL, Tribouilloy C, Grigioni F, Suri RM, Avierinos JF, et al. Twenty-Year Outcome After Mitral Repair Versus Replacement for Severe Degenerative Mitral Regurgitation: Analysis of a Large, Prospective, Multicenter, International Registry. Circulation. 2017 Jan 31;135(5):410-22. doi: 10.1161/CIRCULATIONAHA.116.023340. Epub 2016 Nov 29. PMID: 27899396.

15. Gillinov M, Mick S, Suri RM. The Specialty of Mitral Valve Repair. J Am Coll Cardiol. 2017 Apr 24:S0735-1097(17)30678-2. doi: 10.1016/j. jacc.2017.01.059. Epub ahead of print. PMID: 28476350.

16. Nishimura RA, Otto CM, Bonow RO, Carabello BA, Erwin JP 3rd, Fleisher LA, et al. 2017 AHA/ACC Focused Update of the 2014 AHA/ ACC Guideline for the Management of Patients With Valvular Heart Disease: A Report of the American College of Cardiology/American Heart Association Task Force on Clinical Practice Guidelines. J Am Coll Cardiol. 2017 Jul 11;70(2):252-89. doi: 10.1016/j.jacc.2017.03.011. Epub 2017 Mar 15. PMID: 28315732.

17. Kurfirst V, Mokráček A, Krupauerová M, Canádyová J, Bulava A, Pešl L, et al. Health-related quality of life after cardiac surgery-the effects of age, preoperative conditions and postoperative complications. J Cardiothorac Surg. 2014 Mar 11;9:46. doi: 10.1186/1749-8090-9-46. PMID: 24618329; PMCID: PMC3995816.

18. Goldstone AB, Chiu P, Baiocchi M, Lingala B, Patrick WL, Fischbein MP, et al. Mechanical or Biologic Prostheses for Aortic-Valve and Mitral-Valve Replacement. N Engl J Med. 2017 Nov 9;377(19):184757. doi: 10.1056/NEJMoa1613792. PMID: 29117490.

Надійшла 15.03.2021 